\title{
El Programa de Solidaridad y la organización comunitaria en el
} estado de Morelos, México

\author{
Ana María Chávez Galindo* \\ Francisco Rodríguez Hernández*
}

Este trabajo tiene como finalidad mostrar algunas caracteristicas de las formas de organización de las comunidades que se desarrollaron a partir de la instalación del Programa Nacional de Solidaridad.'

Interesa resaltar el carácter que adquirieron los Comités de Solidaridad ${ }^{2}$ en torno a la organización de la comunidad y a la creación de nuevos liderazgos. Ambos aspectos permitirán conocer nuevas formas desarrolladas por el Estado mexicano para lograr el consenso social de la población, ante los vicios en que habian caido antiguos organismos de control social como la Confederación Nacional de Organizaciones Populares (CNOP), entre otras.

La información de este trabajo se obtuvo con los resultados de la evaluación del Programa de Solidaridad en el estado de Morelos, elaborada en el Centro Regional de Investigaciones Multidisciplinarias (CRIM) de la UNAM (Chávez et al., 1993; 1994); cuyo objetivo consistió en determinar los efectos económico y social de Solidaridad entre la población beneficiada, mediante la confrontación de los resultados de las acciones del programa con los objetivos que se establecian en el mismo. Por tanto, las distintas características de los Comités de Solidaridad presentadas en este traba; son elementos que permiten avanzar en el conocimiento de la nueva dinámica de poder que se establece en el pais.

\section{Introducción}

El instrumento de política social más ambicioso puesto en práctica en México en los últimos años fue el Programa Nacional de Solidaridad. Su objetivo se centró en la lucha contra la pobreza extrema. Sin embargo, al margen de los alcances que tuvo en términos de reducir los rezagos en servicios esenciales y mejorar las condiciones de vida de la población, puede hacerse una interpretación más profunda a partir de las características del programa y la forma en que se desarrolló a lo

* Investigadores del Centro Regional de Investigaciones Multidisciplinarias (CRIM), Universidad Nacional Autónoma de México.

' El Programa Nacional de Solidaridad (Pronasol) fue un programa creado por el gobierno de Salinas de Gortari para combatir las manifestaciones más evidentes de la pobreza extrema.

${ }^{2}$ Los Comités de Solidaridad fueron instancias conformadas por la comunidad. Su tarea consistia en representar a las comunidades demandantes de servicios ante las autoridades responsables del programa para la realización de las obras de Solidaridad. 
largo del sexenio de Carlos Salinas de Gortari, hecho que revela otros objetivos orientados a la estabilidad social y política del país.

En el contexto político del programa, los llamados Comités de Solidaridad adquirieron un papel clave, puesto que eran las células de una nueva organización, pensada para conformar nuevas bases de legitimidad al sistema de gobierno.

En este trabajo presentamos algunas características y expectativas de los Comités de Solidaridad formados en el estado de Morelos entre 1990 y 1992, con el objetivo de exponer elementos que permitan realizar el análisis y evaluación sociopolíticos del Programa Nacional de Solidaridad. Para tal efecto utilizamos datos de una encuesta a dirigentes de Comités de Solidaridad levantada entre enero y febrero de 1993, como parte de la evaluación de Solidaridad en Morelos.

\section{El carácter político de Solidaridad}

\section{Características del programa}

El Programa Nacional de Solidaridad (Solidaridad o Pronasol) ocupaba, en 1992, alrededor de $20 \%$ del total de inversión pública y cerca de $45 \%$ del gasto en desarrollo social (Peón Escalante, 1992: 17). Este programa constituía el cuerpo central de la política social de la administración de Salinas de Gortari. En efecto, según la definición oficial, era el instrumento específico para emprender la lucha contra la pobreza extrema, con cuatro grandes objetivos: 1) mejorar las condiciones de vida de los grupos campesinos, indígenas y de colonos populares; 2) promover el desarrollo regional equilibrado y crear las condiciones para el mejoramiento productivo del nivel de vida; 3) promover y fortalecer la participación y la gestión de las organizaciones sociales y de las autoridades locales; 4) constituir a la solidaridad como una forma permanente de convivencia y relación de los mexicanos y en un vehículo de concertación entre el Estado y la sociedad civil (Programa Nacional de Solidaridad, 1991: 77-83).

De acuerdo con estos cuatro objetivos, se definían como líneas de acción prioritarias la alimentación, la ampliación y mejoramiento de la infraestructura de salud y educación, vivienda y tenencia de la tierra, la electrificación, la procuración de justicia, la infraestructura agropecuaria y la preservación de los recursos naturales. 
Los cuatro principios fundamentales de Solidaridad dan cuenta de la filosofía del programa. Éstos son: 1) respeto a la voluntad, iniciativas y formas de organización de los individuos y comunidades; 2) plena y efectiva participación y organización de las comunidades; 3) corresponsabilidad de los participantes; 4) transparencia, honestidad y eficiencia en el uso de los recursos (Programa Nacional de Solidaridad, 1992: 7).

El primero de los cuatro principios representó la intención de dar respuesta directa y específica a las necesidades de la población tal y como las comunidades las planteaban. Ello implicaba, por un lado, que las comunidades expresaran sus necesidades y contaran con formas efectivas de hacer llegar sus demandas hasta los tomadores de decisiones $y$, por el otro lado, que la toma de decisiones recogiera las prioridades y formas de solución de las necesidades que expresaban las propias comunidades, sin que mediaran criterios diferentes, se impusieran soluciones unilaterales o se decidiera al margen del sentir de los beneficiarios. Si bien a nivel federal y estatal se decide la distribución del gasto a ejercer anualmente, se partía del hecho de que los presupuestos se determinaban a partir del conjunto de gestiones hechas por las comunidades organizadas en los Comités de Solidaridad.

En cuanto a la participación organizada de las comunidades beneficiadas para los trabajos de gestión, administración, financiamiento y ejecución directa de las obras, se buscaba introducir una forma de gestión socializada, así como ser un factor de apropiación -por parte de esta población-de los productos materiales del programa; esto, en ambos casos, puede interpretarse como una delegación de responsabilidades a futuro. El esquema de trabajo de Solidaridad demandaba la participación directa de los beneficiados en la ejecución de las obras o proyectos, organizada esta última a partir de la conformación y operación de los Comités de Solidaridad, instancias representativas de las comunidades beneficiadas.

El principio referido a la corresponsabilidad se refería a la participación, necesariamente coordinada, de instituciones de los tres niveles de gobierno, así como de los beneficiarios organizados en la promoción, financiamiento y realización de los proyectos. Cada proyecto implicaba la celebración de un convenio en donde se especificaban las aportaciones, compromisos y resnonsabilidades de cada una de las partes intervinientes. 
La transparencia, honestidad y eficiencia en el uso de los recursos, el cuarto principio fundamental, se basaba, por un lado, en que las acciones eran puestas en marcha directamente por los beneficiarios, lo cual evitaba desviaciones, por el otro, en el establecimiento de mecanismos de control, supervisión y evaluación de la inversión, que comenzaban con la aprobación de la asignación presupuestal en la Cámara de Diputados.

La característica más relevante del programa era la participación de los beneficiarios con mano de obra, con el financiamiento parcial de las obras y/o con la aportación de materiales. Ahora bien, para que este tipo de participación funcionara efectivamente se requería la organización de la comunidad beneficiada. Dicha organización comenzaba a operar desde el momento en que los miembros de la comunidad se reunían para solicitar su inclusión en el programa. Una vez aprobadas las gestiones para la obra solicitada, la organización debía mantenerse para que los beneficiados se involucraran directamente en la realización de la misma.

Por otra parte, a diferencia del esquema tradicional de designación y aprobación del presupuesto federal para determinados sectores sociales o zonas geográficas, que ha implicado la creación de entidades específicas para diagnosticar y ubicar la problemática en cuestión, Solidaridad no requirió la creación de una entidad pública específica (fuera de la propia Secretaría de Desarrollo Social) para diagnosticar, diseñar las políticas y aun administrar las acciones en las líneas prioritarias, a pesar de la relativa amplitud del conjunto de estas líneas. Con ello se buscó tener coherencia con el adelgazamiento del aparato de Estado, así como con el esquema de participación social y delegación parcial de la toma de decisiones a las comunidades organizadas en los Comités de Solidaridad (Anguiano y Ordóñez, 1994: 70-71).

Pronasol: una estrategia de estabilidad política

Lo que comenzó como un programa asistencial se ha convertido en un principio de gobernabilidad con ideología, organización, recursos, militantes, y objetivos que se extienden mucho más allá del alivio a la pobreza (Dresser, 1992: 55).

Son varios los autores que consideran a Solidaridad como una estrategia política que rebasaba el objetivo general de aliviar la pobreza 
extrema. Cualquier estudioso que haga una reflexión sobre el programa puede advertirlo, pues aspectos como el aparato publicitario implementado o el remarcamiento en la organización comunitaria daban cuenta de ello. La revisión de algunos trabajos de análisis crítico del Pronasol permite ver, en el programa, una estrategia eminentemente política dirigida a asegurar la estabilidad social y la continuidad del sistema de gobierno actual. Estas consideraciones constituyen un referente político más amplio y realista para el análisis de lo observado en el estado de Morelos, en torno a la organización social promovida por el Pronasol. Cabe aclarar que no pretendemos elaborar una argumentación definitiva al respecto, sino plantear elementos analíticos útiles a la temática que nos ocupa.

El programa fue una vía de compensación a los estragos causados en las condiciones de vida de la población por la crisis económica y por la aplicación de las políticas neoliberales. Esto es, las distintas acciones efectuadas bajo este programa permitieron reducir los rezagos acumulados en cuanto a la satisfacción de necesidades esenciales, así como también compensar los efectos negativos por la continuidad de la política económica y prevenir la agudización de los mismos. Por tanto, en la práctica estos aspectos, sin constituir un objetivo explícito del Programa, aseguraban el cumplimiento de los objetivos políticos del mismo.

No obstante, las autoridades estaban conscientes de que para reducir efectivamente los niveles de pobreza era necesario actuar por el lado de sus causas estructurales y no sólo atenuar las carencias en las condiciones de vida de la población; además, consideraban que una elevación del nivel de vida de la población sería producto del desarrollo económico que alcanzara el país en el futuro, a partir de los cambios generados en la economía con su incorporación en el nuevo proceso de globalización.

Por otra parte, interesa destacar que Pronasol fue una política que permitió la supervivencia política del sistema de gobierno, porque buscaba, tanto la legitimación de un régimen que en este aspecto se vio mermado desde un principio, ${ }^{3}$ como lograr un consenso entre los estratos sociales potencialmente explosivos, esto es, los más golpeados

${ }^{3}$ Como es conocido, nunca quedó libre de dudas el triunfo del PRI en las elecciones presidenciales de 1988 . 
por los efectos de la crisis económica y los costos del ajuste neoliberal de la economía.

Se trata, en consecuencia, de un programa pensado para amortiguar los efectos de la crisis y la política económica, y al mismo tiempo, de una estrategia que permite recuperar la imagen del gobierno entre la población que mantiene una oposición latente e incluso abierta (Dresser, 1992).

El Pronasol estaba pensado también como factor esencial para lograr una reestructuración de las bases de apoyo del Estado (Dresser, 1992), en vista del debilitamiento o agotamiento de las bases corporativas tradicionales, ${ }^{4}$ ocasionado por la transformación del modelo productivo y las políticas de ajuste instrumentadas durante los ochenta (Bertranou, 1993: 234). Por tanto, el empeño puesto en la organización comunitaria como fórmula de una nueva relación del gobierno con el pueblo no consistía en la simple idea del respeto a la voluntad e iniciativas de las comunidades, sino en la necesidad de crear un nuevo mecanismo de legitimidad y consenso para el sistema de gobierno.

Adicionalmente, el programa fue pensado como un fondo en el cual la asignación presupuestal se manejara discrecionalmente, de tal suerte que los recursos pudieran orientarse, sin mayor problema administrativo, hacia zonas o grupos sociales en donde el efecto político de la inversión fuera más rentable: el caso de aquellos sectores que mostraban gran descontento hacia el Estado.

En síntesis, con Solidaridad se configuraron nuevos esquemas de "patronazgo" basados en la relación gobierno-beneficiados. Esta modalidad sustituyó al viejo esquema corporativo de distribución de los beneficios y, además, permitió al gobierno recuperar el potencial político que significa la asignación selectiva de recursos financieros hacia determinados sectores de la sociedad (Dresser, 1992; Anguiano y Ordóñez, 1994).

En el contexto anterior, es fácil advertir el papel que tenía la conformación, funcionamiento, capacidad de liderazgo y permanencia de los Comités de Solidaridad. Para el ciudadano común, una parte de las funciones de los comités era claramente observada: la referida a la gestión y ejecución de los proyectos de los cuales se beneficiaban. Pero otra parte no era clara ni para las comunidades ni para los comités mismos: precisamente la que buscaba construir un consenso con el gobierno mediante una nueva organización social.

\footnotetext{
' Vos referimos a los antiguos "sectores" del PRI: obı ero, campesino, popular.
} 


\section{Alcances de Solidaridad en Morelos}

Antes de pasar al análisis del papel adquirido por los Comités de Solidaridad en Morelos, es útil conocer algunos aspectos conclusivos de la evaluación de las acciones desarrolladas con el programa entre 1990 y 1992 (Chávez et al., 1993 y 1994; Chávez y Rodríguez, 1993).

La evaluación de Solidaridad realizada en el CRIM contempló el levantamiento de varias encuestas de opinión que generaron información específica. ${ }^{5}$ Tales encuestas fueron aplicadas a: 1) la población beneficiada por el programa; 2) los directivos de los Comités de Solidaridad; 3) los productores agrícolas beneficiados con los programas de Crédito a la Palabra y Solidaridad para la Producción; 4) los 33 presidentes municipales del estado.

Para fines de evaluación se procedió a agrupar los municipios de Morelos en tres regiones según áreas de influencia de las principales zonas urbanas (Chávez et al., 1994). Se establecieron así las siguientes regiones:

a) Región Norte: integrada por los municipios de Cuernavaca, Juitepec, Temixco, Huitzilac y Tepoztlán. Esta región es la más desarrollada del estado; su población se encuentra en mejores situaciones de ingreso y con menores carencias de servicios públicos.

b) Región Este: se compone de los municipios de Cuautla, Yautepec, Tlalnepantla, Totolapan, Tlayacapan, Atlatlahucan, Yecapixtla, Ocuituco, Tetela del Volcán, Ayala, Jantetelco, Jonacatepec, Temoac, Zacualpan, Tepalcingo y Axochiapan. Esta región es la más pobre del estado, tanto por sus niveles de ingreso como por sus deficiencias de servicios públicos.

c) Región Sur: incluye los municipios de Jojutla, Zacatepec, Puente de Ixtla, Xochitepec, Tlaltizapán, Tlaquiltenengo, Miacatlán, Tetecala, Mazatepec y Coatlán del Río. Se puede caracterizar como zona poco desarrollada, pero está en mejores condiciones que la región Este.

Los resultados de la evaluación mostraron que los alcances de Solidaridad en Morelos no fueron despreciables; se lograron mejoras en las condiciones de vida de poco menos de una tercera parte de la población del estado, pero no puede decirse que se haya logrado una reducción significativa de los niveles de pobreza. Entre 1990 y 1992, Solidaridad logró disminuir en menos de un punto porcentual la pro-

\footnotetext{
${ }^{5}$ Véase el anexo para información sobre la muestra.
} 
porción de población con ingresos de cero a dos salarios mínimos (de 60 a 59\%, aproximadamente). En cambio, el déficit promedio en los servicios de agua entubada, drenaje, electrificación, educación y salud, registró una reducción del orden de $25 \%$ en el mismo periodo.

La dotación de servicios públicos esenciales constituía una prioridad de Solidaridad; sin embargo, no se hizo el mismo hincapié en ciertas problemáticas como la alimentación, el empleo o la productividad de las familias, que si hubieran sido acciones contempladas dentro del programa habrían permitido mejorar efectivamente el nivel de vida de la población. En pocas palabras, las acciones de Solidaridad mejoraron algunos aspectos de las condiciones de vida de la población morelense, pero no atacaron las causas estructurales que son las que originan la pobreza en México.

Por otra parte, la distribución de los beneficios de Solidaridad en Morelos, entre 1990 y 1992, fue desbalanceada, especialmente en los programas de bienestar social, pues se otorgaron en mayor medida en las zonas urbanas, en detrimento de las áreas rurales. En lo relativo a los programas productivos, destinados en su mayoría al sector agropecuario, pudiera pensarse que, por su interés y dirección, se intentaba compensar la menor dotación de servicios en las zonas rurales; pero la evaluación mostró que estos programas tuvieron una efectividad casi nula en términos de elevar la productividad de los campesinos que recibieron estos apoyos. Lo que ocurrió con los programas productivos fue que ellos no tocaban las causas que propician las condiciones marginales del campo, como son el precio de garantía, los mecanismos de comercialización o los bajos rendimientos de los cultivos en tierras de temporal.

Además, la forma como operaba el programa no permitió que los esfuerzos se concentraran en la población más pobre; se beneficiaron, en cambio, algunos grupos poblacionales que no necesitaban este tipo de asistencia.

Tal panorama ocurrió con mayor frecuencia en los programas de infraestructura urbana que se llevan a cabo en colonias populares. En efecto, como la inversión se orientaba a colonias o calles, las familias no pobres, localizadas en lugares donde se realizaban proyectos de Solidaridad, recibían de igual modo los beneficios del programa, aunque en ocasiones no lo requiriesen. En cambio, familias en pobreza extrema residentes en zonas alejadas y con grandes carencias no se incluyeron en el programa. 


\section{Los Comités de Solidaridad en Morelos: conformación, funcionamiento y perspectivas}

Uno de los planteamientos esenciales del Programa Nacional de Solidaridad consistía en lograr un cambio en la organización de la comunidad, de tal suerte que fuera ella misma la que planteara sus necesidades básicas y estableciera las formas de acción, dirección y ejecución para resolverlas.

Este espíritu estaba en la base de la configuración de los Comités de Solidaridad, instancias conformadas e integradas por la propia comunidad, mediante las cuales se intentaba romper con los viejos esquemas de conducción-acción a que ha sido sometida la población durante décadas. Conforme al esquema de participación organizada, los comités podían haberse convertido, por una parte, en los nuevos actores sociales en el ámbito local que condujeran y potenciaran el trabajo de la comunidad para resolver sus problemas inmediatos y mediatos; y, por otra, formaran las nuevas bases de legitimación y consenso del gobierno.

Para saber si los comités adquirieron el papel de nuevos actores en el plano local interesaba conocer tanto la forma y características de su organización y funcionamiento como el tipo de liderazgo ejercido con sus acciones. Con este propósito se analizaron, entre otros aspectos, la participación de la comunidad en su configuración; los antecedentes de organización y liderazgo de los directivos y de la propia comunidad; las características socioeconómicas de los integrantes de los comités en tanto que pudieran ser determinantes en su capacidad directiva; la responsabilidad y participación de sus integrantes en las distintas etapas de ejecución de una acción de Solidaridad; la vinculación con otras instancias de coordinación institucional y la promoción de la organización de la comunidad para la acción. Tal análisis se basa en los resultados de la encuesta realizada a los directivos de Comités de Solidaridad en Morelos que se llevó a cabo a principios de 1993.

\section{Mecanismos de formación del comité}

Un primer aspecto consiste en determinar si había una injerencia directa de Solidaridad en la forma como se organizaba la comunidad; 
en especial, interesa el tipo de intervención que tenía en la formación de los comités. Este hecho es de gran relevancia en tanto que muestra el nivel de autonomía de esta instancia. Para ello se analizaron dos rubros: de qué persona o autoridad surgió la idea de formar el comité y cómo se formó esta instancia. La promoción y nombramiento de estas instancias por autoridades se interpreta como una carencia de autonomía en la comunidad. En cambio, si la promoción y la constitución fue desarrollada por la propia comunidad se estaría hablando de capacidad e independencia para organizarse.

En términos generales se aprecia que la formación e integración de los comités se desarrollaba de manera autónoma, pues casi no intervenían los responsables de Solidaridad o las autoridades locales. Así, en relación con la idea de formar el comité, ésta provino en la mayoría de los casos (55\%) de la propia comunidad, sea de los mismos colonos, los vecinos o las juntas de padres de familia en las escuelas, independientemente del año de creación del comité. El promotor de Solidaridad propuso la formación de esta instancia en $31 \%$ de casos; aunque fue a partir de 1991 cuando desarrolló con mayor intensidad esa labor. Los agentes externos tales como autoridades, dirigentes del Partido Revolucionario Institucional (PRI) o alguna otra persona (los maestros en las escuelas, por ejemplo) tenían una injerencia menor ( $14 \%$ de los casos) (gráfica 1 ).

Lo declarado por los directivos en relación con el mecanismo de formación del comité reafirma la autonomía de la comunidad en este aspecto: la mitad (51\%) de los dirigentes entrevistados indicó que el comité se formó mediante una asamblea; poco menos de una tercera parte $(31 \%)$ señaló como mecanismo la elección con voto secreto; más de una décima parte $(11 \%)$ informó que había sido por nombramiento de autoridades, ya sea estatales o municipales; y el resto $(8 \%)$ opinó que fue por nombramiento del promotor (gráfica 1). Esto significa que en $80 \%$ de los casos la comunidad procedió a conformar la instancia organizativa que la vincularía con Solidaridad sin la injerencia o conducción de agentes externos a ella. Hay que aclarar, sin embargo, que el contar con esta instancia era un requisito indispensable para llevar a cabo una acción de acuerdo con el programa de Solidaridad. Sin embargo, se aprecia la posibilidad y capacidad que tienen las comunidades para organizar y conformar sus propias instancias de dirección-acción.

La situación antes reseñada es válida para el conjunto de comités de Morelos, pero muestra diferencias según regiones. Contrastan las 
GRÁFICA 1

Formación del Comité de Solidaridad

Quién tuvo la idea de formar el comité

Miembros de

la comunidad

$55.1 \%$

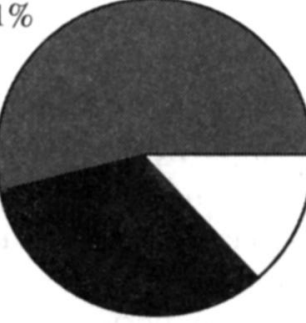

Promotor de

Solidaridad $31.1 \%$
Agentes externos $13.8 \%$

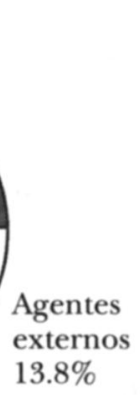

Fuente: Encuesta a directivos de comités.
Mecanismo para la

formación del comité

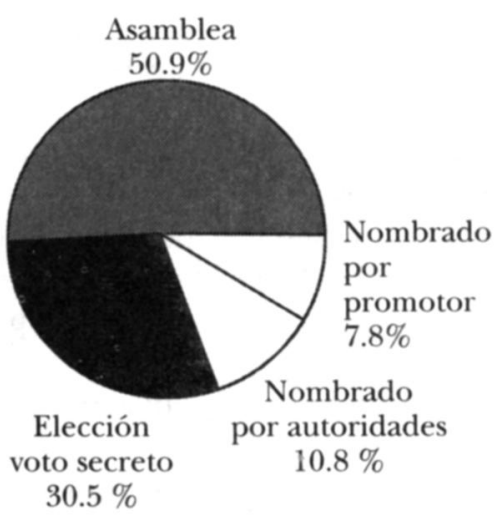

distintas modalidades seguidas para la elección de comités en la región Norte, la más desarrollada, contra lo encontrado en las menos desarrolladas. ${ }^{6}$ Mientras que en la primera las asambleas constituían el mecanismo mayormente empleado para la elección de los integrantes del comité ( $76 \%$ de los directivos respondieron en este sentido); en las regiones atrasadas tal procedimiento fue empleado sólo en $28.6 \%$ de los casos. Asimismo, en estas últimas regiones se distingue la presencia del promotor en el nombramiento del comité, pues este proceder se registró en una cuarta parte (26.5\%) de los casos. En cambio, en la región desarrollada el promotor intervino sólo en $2.8 \%$ de los casos.

\section{Antecedentes de organización y liderazgo}

Solidaridad promovía el surgimiento de nuevos líderes en la comunidad al incorporar en los comités a población sin experiencia organizativa

${ }^{6}$ Consideramos como zona desarrollada a los municipios que conforman la región Norte y las zonas menos desarrolladas comprenden los municipios de las regiones Este y Sur. 
previa, pero dispuesta a asumir las responsabilidades en torno a la organización de la comunidad.

En efecto, los resultados de la evaluación mostraron que entre los dirigentes de estas instancias, muy pocos habían ejercido previamente funciones de liderazgo entre la población. Al respecto, tres de cada cuatro dirigentes de Morelos $(\mathbf{7 5 \%})$ indicaron que no habían tenido experiencias previas de organización (gráfica 2). Esta situación es más pronunciada entre los grupos tradicionalmente marginados de las instancias y/o espacios de dirección-acción, como son los dirigentes del sexo femenino o los que residen en el medio rural, ${ }^{7}$ por señalar algunos casos: $82 \%$ de las mujeres dirigentes y $79 \%$ de los dirigentes del medio rural indicaron que su participación en el comité constituía su primera experiencia de organización y dirección.

Ahora bien, la población que había tenido una experiencia previa manifestó que fue en el PRI o en organizaciones sociales no partidarias o no corporativas (gráfica 2). La participación en organizaciones sindicales o en organizaciones de colonos fue reducida, y mínima

\section{GRÁFICA 2}

Antecedentes de organización de los directivos de comités

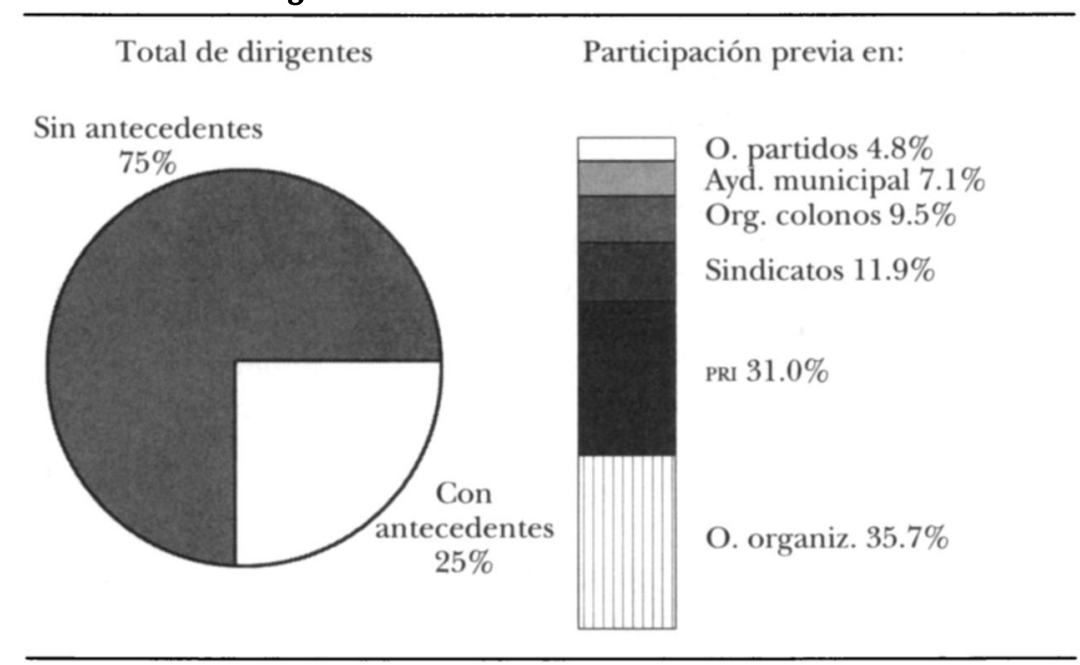

Fuente: Encuesta a directivos de comités.

${ }^{7}$ Se considera población rural la que reside en localidades menores de 2500 habitantes. 
la participación en otros partidos políticos. Destaca, asimismo, el hecho de que entre los hombres la experiencia organizativa más frecuente fue en el PRI (32\%); en segundo término se encuentran otras organizaciones sociales no partidarias $(29 \%)$. En cambio, la mujer que había participado previamente lo hizo en organizaciones sociales (50\% manifestó esta dirección) y un porcentaje más bajo estuvo en el PRI ( 29 por ciento).

Este panorama nos muestra diferencias sustanciales entre hombres y mujeres en el tipo de organización del que han formado parte con anterioridad, hecho que puede traducirse en un comportamiento distinto ante la comunidad. En efecto, por el carácter de cada una de ellas -el PRI, una organización partidaria; las organizaciones sociales, agrupaciones de individuos con o sin filiación partidaria que luchan por demandas muy concretas- la experiencia organizativa adquirida es diferente, lo que se traduce en distintas formas de trabajar en la organización de la comunidad. Con esta consideración y tomando en cuenta la elevada participación de la mujer en las organizaciones sociales puede decirse que, si bien su participación organizada fue menos frecuente que la del hombre, ésta fue más rica en términos de involucrarse con la comunidad para la solución de sus problemas.

Por otra parte, entre los directivos que tienen antecedentes organizativos, resalta la pertenencia que mantienen con las organizaciones anteriores: dos de cada tres dirigentes $(67 \%)$ indicó que continuaban en ellas, sea en un sindicato, una organización social o un partido político. Asimismo, una parte importante de estos directivos (64\%) ha tenido un cargo dentro de dichas organizaciones. La mayoría de esos cargos han sido puestos directivos o de mando: $41 \%$ se había desempeñado como presidente; $33.3 \%$ como secretario o tesorero; y como vocal 26 por ciento.

Esta experiencia previa en la conducción de organizaciones se podía convertir en un elemento favorable al momento de participar como dirigentes en los Comités de Solidaridad. Sin embargo, tal circunstancia se presentó en tan pocos casos que su impacto fue mínimo.

Por otro lado, independientemente de la experiencia previa de los directivos, la existencia de liderazgos en la comunidad antes de la configuración de los comités y su posible incorporación a esta instancia, constituye otro aspecto importante para este análisis, pues permite conocer si Solidaridad propició el surgimiento de nuevos líderes o si 
las nuevas estructuras organizativas se conformaron con los antiguos líderes de la comunidad.

La existencia previa de líderes en la comunidad puede significar estar frente a una comunidad que cuenta con una o varias personas que, por su trabajo organizativo, se hayan ganado el reconocimiento como conductores en la lucha por resolver sus problemas. En tal situación, incorporar a estos líderes a los comités podría garantizar un mejor desempeño en la organización de actividades que benefician a la comunidad. No obstante, si éste fuera el caso, la promoción de nuevos líderes pudiera no darse y, por tanto, no se cumpliría uno de los principales objetivos del programa.

En cambio, la ausencia previa de líderes en la comunidad -ya sea porque la población no ha dado pasos importantes hacia su organización, o porque las personas o grupos que trabajan con esta perspectiva no han logrado un reconocimiento en la comunidad- pudieron implicar un mayor trabajo organizativo de Solidaridad para transformar la dinámica de la comunidad; sin embargo, de haberlo logrado hubiera sido de gran beneficio para los habitantes.

La información que proporcionaron los directivos de comités sobre la existencia de líderes indica que son muy pocos los dirigentes reconocidos por la población. Sólo en $20 \%$ de los casos se mencionó que la comunidad contaba con líderes antes de la formación de los comités (gráfica 3). Éstos, además, surgieron mayormente en las zonas más desarrolladas del estado: la región Norte, en donde $25 \%$ de los dirigentes consignó la existencia de líderes previamente; en cambio, en la región Este (la menos desarrollada) sólo en $16 \%$ de los casos se mencionó tal situación.

Destaca, sin embargo, que a pesar de la existencia de líderes en las comunidades, éstos han tenido una participación reducida en los comités. Del total de comunidades donde había líderes, sólo en $35 \%$ de los casos se incorporaron a dicha instancia (gráfica 3). Tal situación se presentó con mayor frecuencia en la zona más desarrollada, donde $44 \%$ de los antiguos líderes eran miembros de los comités. En las regiones más atrasadas, sólo una cuarta parte de los líderes se encontraba en estas nuevas instancias de organización. Por último, cabe destacar que la escasa participación de antiguos líderes en los Comités de Solidaridad pudiera explicarse por la cantidad de acciones que debían efectuar al momento de incorporarse a dicha dependencia y no por un rechazo de la comunidad. Esta última opción sólo fue aducida en $15 \%$ de casos. 
GRÁFICA 3

Existencia de líderes antes de Pronasol e incorporación en los comités

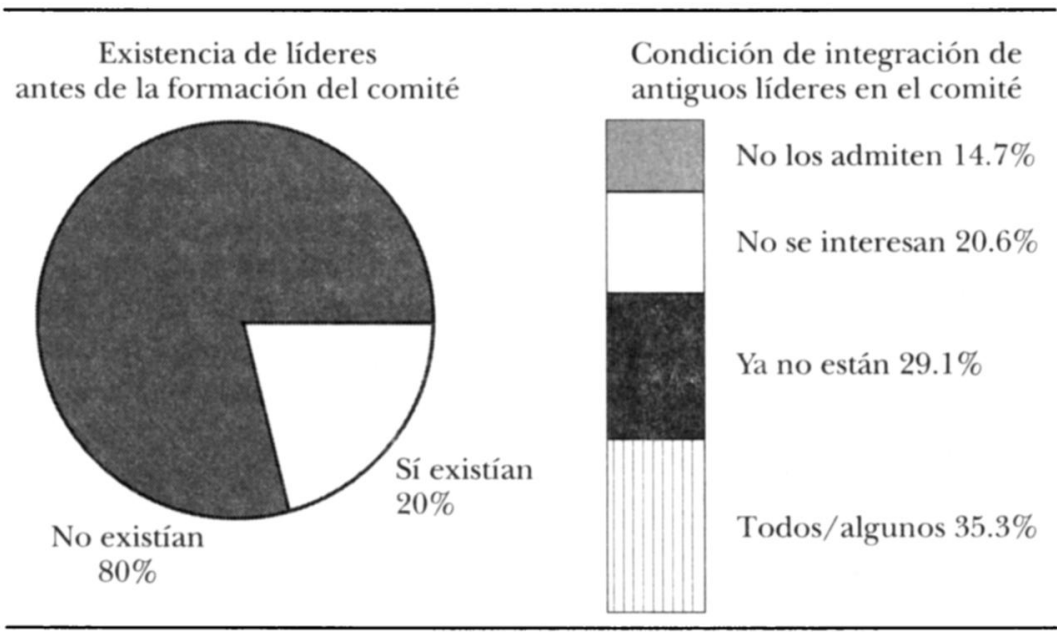

Fuente: Encuesta a directivos de comités.

Otro hecho que puede constituir un elemento importante en la organización de la comunidad se refiere a la existencia de personas y/o grupos que han estado trabajando por el desarrollo comunitario, independientemente de que se les reconozca liderazgo local. Al respecto, destaca que, para el total de la entidad, en $37 \%$ de casos se mencionó que sí existía esta figura orgánica (gráfica 4). Sin embargo, al igual que lo señalado respecto a los líderes, en la región de mayor desarrollo fue más frecuente la presencia de grupos o personas que trabajaban por la comunidad: $52 \%$ de los directivos de la región Norte hizo tal señalamiento, mientras que en la región Este, sólo en $22 \%$ de los casos se presentó dicha situación.

Pero la presencia en las comunidades de personas o grupos que desarrollaban acciones en beneficio de la comunidad, tal vez no tenga un gran significado en términos de enseñanza para el nuevo esquema de organización, pues la mayoría de estas personas o grupos no formaban parte de organización alguna, como lo manifestó $59 \%$ de los directivos; $y$, entre las personas o grupos que sí habían formado parte de una estructura organizativa, el PRI fue la principal instancia en la que participaron (gráfica 4). 
GRÁFICA 4

Persona y/o grupo que organizaba la comunidad antes de Solidaridad

Opinión del total de directivos

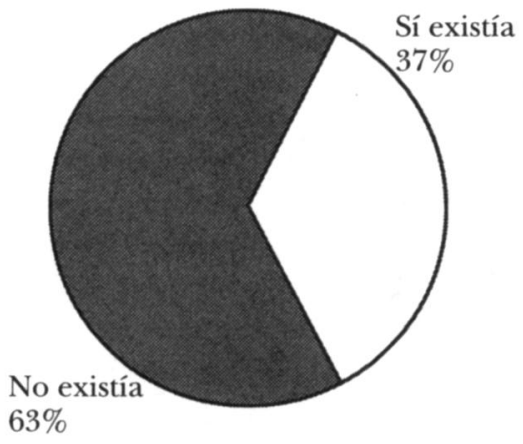

Tipo y forma de participación

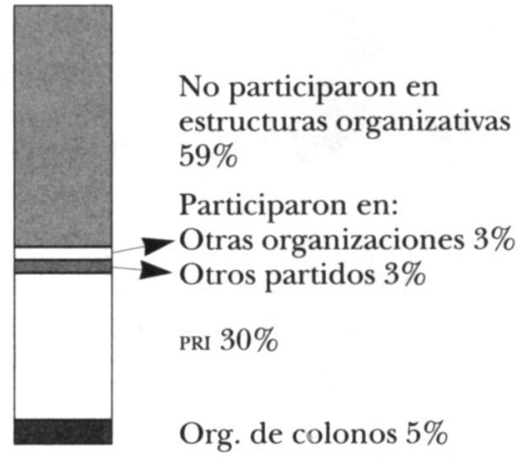

Fuente: Encuesta a directivos de comités.

Características de los directivos

En cuanto a las características socioeconómicas de los integrantes de los comités, destaca que, en términos generales, son distintas a las que tiene el conjunto de la población beneficiada, lo que en buena parte explica por qué fueron elegidos para representar a la comunidad. Hay que destacar los siguientes aspectos:

- Mayor escolaridad que la del conjunto de la población beneficiada: entre los directivos de comités en el estado de Morelos, $28 \%$ no tenía estudios o cursó sólo algunos años de primaria sin concluir este nivel educativo; entre la población beneficiada $34 \%$ se encontraba en la misma situación. ${ }^{8}$

- Una elevada participación en la actividad económica, en especial entre las mujeres: $68 \%$ de los directivos declaró realizar una actividad económica; entre los directivos del sexo femenino $42 \%$ trabaja,

${ }^{8}$ Información de la Encuesta a Población Beneficiada por Solidaridad en el Estado de Morelos (Chávez et al., 1993). 
porcentaje superior en $67 \%$ al que registró el total de mujeres beneficiarias.

- Mayor ingreso que el total de beneficiados como reflejo de su mayor escolaridad y del tipo de actividad económica que desarrollaban: entre los directivos que declararon percibir ingresos, aproximadamente una tercera parte (38\%) recibía hasta dos salarios mínimos; en cambio, en la población beneficiada más de la mitad (54\%) se encontraba en este rango de salarios mínimos. No obstante, hay claras diferencias en los niveles de ingreso de los dirigentes según género o ámbito geográfico: poco más de la mitad $(54.5 \%)$ de las mujeres directivas tenía hasta dos salarios mínimos, mientras que en esta situación se ubicaba menos de la tercera parte $(30 \%)$ de los hombres. Estas mismas proporciones se registran en el medio rural y en el urbano, respectivamente.

- Se presenta una participación casi igualitaria de hombres y mujeres entre los dirigentes de los comités, aunque en las áreas urbanas o en las más desarrolladas, fue mayor la participación de la población femenina.

- Se trata, en casi todos los casos, de población madura: la edad media de los dirigentes masculinos era de 48 años, en tanto que para las mujeres, de 37 años. Tal característica expresa que eran elegidas aquellas personas que infundían respeto entre la comunidad.

- Precisamente por la responsabilidad otorgada al puesto de dirigente del Comité de Solidaridad, la mayoría de las personas elegidas eran jefes de hogar $(56.3 \%)$ o cónyuges del jefe (38.9 por ciento).

En suma, la combinación de estas características está acorde con la visión de que se elegían como directivos a aquellas personas que pudieran asegurar responsabilidad en la conducción de esta instancia, con base en un reconocimiento directo de necesidades y la aceptación del compromiso y corresponsabilidad de realizar y dar seguimiento a los múltiples trámites administrativos necesarios al efectuar una obra de Solidaridad.

Estructura, composición y temporalidad de los comités

Otros aspectos a destacar respecto a los comités tienen que ver con su estructura formal, su composición real y su actuación, pues permiten apreciar la incidencia que tuvieron los comités en la organización y participación de la comunidad. 
El Programa de Solidaridad establecía que los comités deberían estar integrados por un presidente, un secretario, un tesorero y tres vocales; entre estos últimos, un puesto era de control y vigilancia, cuya función principal consistía en vigilar la transparencia de recursos y el uso adecuado de ellos. Aunque este esquema de integración fue seguido por todos los comités, en la práctica las actividades se concentraron en dos o tres personas a lo sumo. Las personas que se mantuvieron activas dentro de los comités tendieron a ser las que ocupaban los cargos principales: presidente, tesorero y/o secretario. Esta situación se relaciona posiblemente con el bajo interés que tenía la comunidad para organizarse, aspecto que se tradujo en una falta de continuidad de acciones dentro del programa, una vez satisfecho el objetivo que dio origen al comité.

Por otra parte, de la distribución por sexo observada en los distintos cargos de esta instancia, se aprecia que, no obstante la alta participación de la mujer, se reprodujo el esquema tradicional acerca de quién toma las decisiones: los varones continuaron siendo la mayoría en casi todos los cargos (del total de presidentes de comités, secretarios, tesoreros o vocales de control y vigilancia, $57,53,52$ y $56 \%$, respectivamente, eran del sexo masculino; la mujer participó con mayor frecuencia en el cargo de secretaria, tesorera o vocal). Este dominio hizo difícil la incorporación de la mujer a las tareas organizativas que potenciaran el trabajo comunitario.

Un segundo aspecto a contemplar dentro de este apartado es el referente al momento de formación de los comités y el tiempo que se mantuvieron vigentes, pues permite detectar la congruencia entre las acciones desarrolladas y los objetivos del programa, especialmente aquellos sobre la organización social.

La constitución de esta instancia se inició con el Programa de Solidaridad desde 1989, como órgano mediador entre la comunidad y las autoridades para la realización de las obras. Ahora bien, la información proporcionada por los directivos de los comités indicó que su formación se desarrolló paulatinamente y de manera diferencial: en un primer momento, se establecieron en las zonas desarrolladas del estado, en las zonas deprimidas la creación de comités fue posterior, acentuándose en el último año evaluado. El significado de este panorama, visto a nivel regional, es que muy posiblemente se formaron comités con mayor facilidad donde ya había bases de organización de la comunidad; situación que no se observó en el medio rural caracterizado por su dispersión y desorganización. Por tanto, Solidaridad no propi- 
ció, como hubiera debido y sobre todo al inicio del programa, la formación de instancias de dirección y organización en los lugares donde más se requería.

Por otro lado, con la finalidad de apreciar la permanencia y continuidad de los comités en su trabajo para la solución de los diversos problemas de la comunidad, se consignó información sobre el número de obras desarrolladas. Al respecto, se observa que en $57 \%$ de los casos, el comité promovió al menos dos obras. Sin embargo, $61 \%$ de estas obras adicionales pertenecía al Programa Escuela Digna, cuyo impulso fue promovido por los directivos de las escuelas más que por el comité. Esta situación pone en evidencia el corto tiempo que duraba en funciones la mayoría de los comités. La tónica general fue constituirse para ejecutar una obra y, al concluirla, disolverse. Tal hecho hacía difícil consolidar la incipiente organización de la comunidad y crear un liderazgo con nuevos e inexperimentados dirigentes.

\section{Funcionamiento de los comités}

La frecuencia de las reuniones internas de los comités -y de éstos con la comunidad- así como el carácter de las personas que conducían las reuniones, permitió apreciar la vida, organicidad y autonomía de éstos y el grado de compenetración con la comunidad.

De lo observado acerca del funcionamiento de los comités se puede concluir que se dieron pasos importantes en la manera de organización de las comunidades y las acciones que desarrollaban sus dirigentes, pero los avances no fueron homogéneos.

Particularmente, en la región Norte se observó mayor autonomía y democracia en la constitución e integración de los comités. Ello no ocurrió en las regiones más deterioradas del estado (Este y Sur), donde la comunidad no ha logrado consolidar una capacidad de organización que le permita actuar sin el apoyo o concurso de agentes externos a ella misma. Las repercusiones de esta falta de organización propia se perciben, por un lado, con la presencia de elementos ajenos a la comunidad que promueven su organización, y por otro, en las carencias de los satisfactores básicos que tiene esta población y en lo poco que obtuvieron con el Programa de Solidaridad.

En lo relativo a la frecuencia de las reuniones internas del comité, y de éste con la comunidad, cerca de la mitad de los dirigentes in- 
formó que éstas se llevaban a cabo sólo cuando había un asunto a tratar. Esto significa que no había una comunicación continua entre el comité y la población, aspecto básico cuando se pretende transformar las formas organizativas de la sociedad.

La independencia que guardan los comités respecto de las autoridades se analizó también a partir de la persona que conducía las asambleas. Las respuestas de dos de cada tres dirigentes masculinos indican que los mismos miembros del comité guiaban las reuniones. Sin embargo, en una décima parte de los comités, las autoridades municipales o los directivos de las escuelas eran las personas que llevaban la conducción de las mismas.

En relación con el número de personas que asistían a las asambleas convocadas por el comité, aspecto que permite apreciar su grado de movilización y presencia del comité en la comunidad, la información de los dirigentes mostró que el promedio de asistentes era de 81 personas, cantidad significativa en términos de influencia. No obstante, cuando se revisa esta información según los programas, se distingue que sólo en el Programa Escuela Digna se presenta un número elevado de asistentes. De acuerdo con las características de ese programa, puede decirse que la movilización no es atribuible al comité, sino más bien a los llamados que realizaban los directores de las escuelas. ${ }^{9}$ En los programas de urbanización o los de tipo asistencial, el promedio de personas que acudían a las asambleas fue inferior a 50, de lo cual se infiere que la influencia directa que tenían los comités la ejercían hacia un grupo más reducido de beneficiados. ${ }^{10}$

Por otra parte, el desconocimiento que expresaron algunos dirigentes de comités en lo relativo a los costos de las obras y a las aportaciones monetarias o materiales de la comunidad para la obra, muestra el bajo compromiso que persistía en algunos casos, donde se integraban al comité para cumplir con la formalidad que exigía Solidaridad pero no desarrollaban una función real de dirección, gestión y organización de la comunidad.

${ }^{9}$ El Programa Escuela Digna está dedicado a la reparación y mantenimiento de los inmuebles escolares. Durante el levantamiento de la Encuesta a Directivos de Comités se observó cierto grado de manipulación de los comités por parte de los directores de las escuelas. No se documentó la magnitud de esta situación.

${ }^{10}$ Los Programas de Urbanización evaluados fueron Pavimentación, Agua Potable, Drenaje, Electrificación y Alumbrado Público. Los Programas Asistenciales fueron: Niños de Solidaridad y Mujeres en Solidaridad. 


\section{Eficacia de Solidaridad y su impacto en la comunidad}

Uno de los grandes logros de Solidaridad fue precisamente la eficacia de las acciones emprendidas. Así, al contrario del panorama que se presentaba anteriormente a las demandas de la comunidad, con Solidaridad los problemas se resolvieron con mayor rapidez y eficacia según lo manifestó $84.4 \%$ de los directivos. De acuerdo con los dirigentes, ello fue producto de la participación de la población en la organización conformada para solicitar y ejecutar acciones de acuerdo al programa: tres de cada cuatro dirigentes así lo expresaron. En segundo término, las gestiones y trámites se agilizaron, lo cual se expresó en una respuesta rápida a lo solicitado: por esta vertiente se inclinó uno de cada cuatro directivos.

Cuando los directivos expresaban que con Solidaridad no se habían resuelto eficazmente las demandas de la comunidad, los dirigentes opinaban que ello se debía, entre otros aspectos, a la falta de participación de la comunidad $(46.2 \%)$ y a conflictos políticos entre los miembros de la misma (26.9\%). Ambos hechos impidieron la formación de un frente sólido y numeroso que llegara a acuerdos sobre las demandas esenciales y exigiera el apoyo y los medios para su solución.

Por otra parte, así como se expresaron favorablemente los dirigentes de los comités a la solución de sus demandas con Solidaridad, en el mismo sentido era su opinión sobre los beneficios para la comunidad; esto es, en lo relativo al ingreso de las familias, la salud de la comunidad, la seguridad de los colonos, la revalorización de las propiedades de la comunidad y, principalmente, la participación de la comunidad. Casi la mitad de los dirigentes $\mathbf{( 4 9 . 1 \% )}$ consideró como muy alto el impacto del programa en la comunidad y cerca de un tercio $(30.5 \%)$ señaló que el impacto había sido alto. Esto significa que cuatro de cada cinco dirigentes opinó que Solidaridad repercutió favorable o muy favorablemente en la comunidad, mejorando de manera significativa sus condiciones de vida. Esta opinión exhibe algunas variantes según las diversas características de los dirigentes. Así, por ejemplo, más de la mitad de los dirigentes del medio rural o los de las regiones menos desarrolladas del estado tuvo una opinión más favorable del programa que los de zonas urbanas en general o de la región Norte. Estas diferencias se pueden explicar tal vez por la percepción que tiene la población sobre la pobreza y las acciones que se desarrollan para combatirla. Pequeñas acciones en localidades muy deterioradas tienen un efecto visible más importante que grandes accio- 
nes en poblaciones que han satisfecho en mayor grado sus necesidades vitales.

Asimismo, se observa que las opiniones más favorables hacia Solidaridad fueron expresadas por directivos de comités que mantenían una actividad organizativa continua, a diferencia de lo que expresaron aquellos que sólo se reunían cuando había un asunto por tratar. Igualmente, la opinión fue positiva cuando había expresiones de autonomía en los comités, como por ejemplo las asambleas convocadas y conducidas por los mismos miembros de estas instancias.

En general, lo expresado por los dirigentes muestra el esfuerzo desplegado por los comités para llevar a cabo las tareas a las que se habían comprometido de manera lo más satisfactoria posible. Cuando su desempeño no había sido tan bueno como hubieran querido, opinaban que se debía a su falta de experiencia, de organización y de participación de la comunidad.

\section{Reflexiones finales}

Hay tres aspectos que destacan como objetivos políticos implícitos del Programa de Solidaridad:

1) Amortiguar los efectos negativos en las condiciones de vida de la población por la política económica neoliberal, por medio del denominado "combate a la pobreza".

2) Recuperar la valoración popular del gobierno vía el consenso de la comunidad.

3) Conformar una nueva estructura de organización social, cuya célula sería el Comité de Solidaridad; nueva base de sustento al sistema que sustituiría las ya rebasadas estructuras corporativas tradicionales.

Los resultados de la evaluación del Pronasol en Morelos mostraron el cumplimiento de estos objetivos implícitos, lo que pone en evidencia el fin político de Solidaridad como una clara estrategia de gobernabilidad. Pero, en cuanto a la organización social y a la creación de nuevas redes de poder local, tema central del presente trabajo, la evaluación puso en evidencia que poco se avanzó en la conformación de la organización permanente de la comunidad.

En relación con el combate a la pobreza extrema, es evidente que el programa tuvo un efecto real en términos de mejorar algunos aspectos de las condiciones de vida de la población beneficiada, como 
lo indica la reducción de una cuarta parte del déficit en servicios públicos esenciales en sólo tres años. Asimismo, la cobertura que alcanzó fue importante: en Morelos aproximadamente uno de cada tres habitantes recibió algún beneficio de Solidaridad.

El Pronasol, sin embargo, no ha sido capaz de reducir los niveles de pobreza, porque no atiende a sus causas estructurales. Así, el porcentaje de población bajo la línea de pobreza, que en Morelos abarcaba $60 \%$ de la población en 1990 , se redujo en menos de $1 \%$, entre ese año y 1992.

Además de los magros logros en el combate a la pobreza extrema, la distribución de los recursos fue desigual, pues dio preferencia a las zonas más desarrolladas del estado. Tanto la distribución de la inversión cuanto la opinión de los beneficiarios, pusieron en evidencia que las posibilidades de las comunidades para integrarse al programa estaban en relación directa con el grado de acceso a las instancias de gestión y decisión de Solidaridad: las comunidades rurales y alejadas de las principales ciudades recibieron la menor atención, mientras que las urbes fueron las más beneficiadas, tanto en términos absolutos como relativos.

El programa contribuyó efectivamente a mejorar la imagen del gobierno entre la población y avanzó en el consenso requerido para mantener la estabilidad social y la continuidad del sistema, como se deduce de los resultados de las elecciones locales y federales de 1994, favorables al PRI. En pocos lugares la población beneficiada tuvo una opinión desfavorable hacia Solidaridad, aun en aquellos donde se prefiere al Partido de la Revolución Democrática (PRD). Este consenso, sin embargo, estaba asociado con la imagen que se difundió de Salinas de Gortari, más que con el aparato de gobierno en su conjunto o con el PRI.

Por otro lado, Solidaridad propició y facilitó la organización de la comunidad para solucionar carencias importantes de satisfactores básicos. Ello se expresa tanto por la adopción de la estructura organizativa requerida para llevar a cabo una obra conforme al programa, como por la presión ejercida para exigir el cumplimiento de sus demandas básicas, o bien por la colaboración con dinero, materiales o trabajo para la ejecución de la acción emprendida.

En efecto, para la realización de las distintas obras, la comunidad configuró, sin problema alguno, la estructura organizativa requerida: los Comités de Solidaridad. Con la constitución de estas instancias se fomentó la autonomía de la comunidad, al dejar que ella misma buscara la forma y estableciera los procedimientos para nombrar a sus di- 
rigentes, quienes respondieron la mayoría de las veces a las expectativas de la comunidad por resolver necesidades específicas.

Sin embargo, es necesario matizar el éxito de Solidaridad en lo relativo a la organización de la comunidad y a la conformación de los comités pues este procedimiento fue inducido; constituía un requisito indispensable del programa para que las demandas específicas pudieran ser tomadas en cuenta y se asignaran los recursos financieros necesarios para su realización.

Otro elemento que reduce los logros de Solidaridad como estrategia para consolidar la organización de la comunidad tiene que ver con el carácter de las demandas que atendía este programa. Al ser éstas tan concretas y limitadas, la experiencia organizativa de un comité se reducía sólo al lugar donde se llevaba a cabo la obra y al tiempo que tardaba en efectuarse; no había razón tangible para sostener la existencia de esta instancia una vez resuelta la necesidad. Poco hacían los comités por ampliar su ámbito de influencia hacia otros espacios de la comunidad y permanecer en constante actividad, aspectos necesarios si se pretende incidir en la organización comunitaria.

El comité, en realidad, era la instancia o la estructura adoptada por la comunidad como requisito para la realización de la obra. Tuvo, sin embargo, la función política de lograr el consenso de la comunidad con el gobierno porque por su conducto la población se sintió representada ante el mismo. La satisfacción de las demandas de la comunidad y la incorporación de la población directamente afectada, por medio de una organización más aparente que real para la realización de las obras correspondientes, fue la estrategia seguida por el gobierno para conseguir el consenso de la población. Mediante esta forma, la mínima atención a las demandas comunitarias por parte del gobierno era magnificada por una población tradicionalmente marginada de cualquier acción gubernamental.

Por cuanto a la formación de nuevos liderazgos en la comunidad, la evaluación para Morelos puso en evidencia sus limitaciones.

La dirección de la satisfacción de las demandas de la población de Morelos mostró una correspondencia directa hacia lugares donde había una capacidad de organización mayor, sea por la existencia de antiguos líderes y su incorporación a Solidaridad, sea por la experiencia adquirida en los muchos años de lucha y organización. La atención brindada a la población de la región Norte es prueba de ello.

Ahora bien, en el Morelos de hoy existen líderes en algunas comunidades, pero pocos se incorporaron a los comités; lo hicieron 
aquellos que tenían vínculos directos o mediados con instancias gubernamentales. Los que han realizado un trabajo comunitario al margen del gobierno poco creyeron en estas nuevas formas organizativas y menos fueron convocados a formar parte de ellas. Por eso fue frecuente la respuesta de los dirigentes entrevistados de que había inexperiencia en la conducción de acciones organizativas comunitarias antes de Solidaridad. Desde este punto de vista, el Pronasol tenía entonces la posibilidad real de propiciar el surgimiento de nuevos líderes y romper con las viejas estructuras organizativas. Sin embargo, esta posible capacidad de liderazgo de los nuevos dirigentes para organizar la comunidad se vio limitada por el corto tiempo que duraban las actividades de un comité (ante el escaso número de obras que realizaba y el poco tiempo que demanda la ejecución de una obra) y por el ámbito de acción reducido (una calle, una colonia, una escuela). Por tanto, difícilmente, en tan corto tiempo y con tan limitada experiencia organizativa, el comité y sus integrantes pudieron transformar la dinámica de la comunidad y funcionar como nueva instancia de poder local. Este poder sigue permaneciendo ligado y controlado por las autoridades federales, estatales o municipales. Para muestra es suficiente apreciar el dominio que aún mantiene el ayuntamiento local. No se niega, sin embargo, que se hayan abierto nuevos canales de organización y dirección de los recursos federales. Pero aún no se aprecian cambios radicales.

La creación de una organización comunitaria diferente deberá partir de nuevas relaciones entre pueblo y gobierno, en donde la participación popular tenga un peso concreto y absoluto. Esta propuesta no podía provenir de Solidaridad puesto que, en el fondo, perseguía mantener el control de los líderes y las organizaciones sociales.

En conclusión, los efectos reales del Pronasol en el grado de pobreza en Morelos, así como en la organización social, pusieron en evidencia la distancia entre el discurso y los objetivos reales del programa. No importa tanto reducir la pobreza como convencer a la población de que el gobierno hace algo para mejorar sus condiciones de vida. Pronasol, sin duda, fue una estrategia de gobernabilidad.

\section{Anexo}

Para la evaluación del efecto social de Solidaridad se levantaron cuatro encuestas de opinión dirigidas a la siguiente población: 
1) Hogares beneficiados.

2) Productores agropecuarios beneficiados con los programas Crédito a la Palabra y Fondos de Solidaridad para la Producción.

3) Directivos de Comités de Solidaridad.

4) Presidentes municipales.

La encuesta a hogares beneficiados tuvo un diseño muestral de tipo probabilístico polietápico que permitió obtener resultados con precisión estadística para la población beneficiada con un nivel de confianza de $80 \%$ en el nivel de las regiones. Las etapas de selección fueron: a) selección de municipios dentro de cada región; b) selección de localidades dentro de los municipios; $c$ ) selección de subprograma y obra.

Se utilizó el monto de gasto ejercido en Solidaridad como ponderador de la probabilidad de selección en las primeras dos etapas. En la tercera se desagregó en el nivel de obras y se buscó equilibrar el número de entrevistas por programa. Esta etapa incluyó la verificación en campo de las obras seleccionadas y su localización física, de manera que siempre se asegurara la cobertura de la muestra.

Con niveles de confianza de $80 \%$, un nivel máximo de no respuesta de $15 \%$ y un multiplicador de 2.3 adicional por efecto de diseño, se estimó un tamaño de muestra de 326 hogares para cada una de las tres regiones. El tamaño de muestra estatal de 978 viviendas permitió tener para los mismos márgenes de error ya señalados, una confianza de 92 por ciento.

Para las Encuestas a Productores Agropecuarios y Comités de Solidaridad se determinaron tamaños de muestra de 181 para el primer caso y de 169 para el segundo. En el caso de los comités, se realizó un muestreo aleatorio simple sobre un directorio de 2552 comités. Para la Encuesta a Productores, se realizó el muestreo con el cierre de ejercicio de los programas Crédito a la Palabra y Fondos de Solidaridad para la Producción. Ambas encuestas se diseñaron para obtener representación estadística a nivel estatal. El diseño de los cuestionarios se basó en la metodología e intrumentos diseñados por El Colegio de la Frontera Norte y El Colegio de México.

La encuesta de productores agropecuarios se complementó con entrevistas a los directivos de los comités encargados de distribuir los créditos o apoyos monetarios. En el caso de Crédito a la Palabra, se entrevistaron a directivos de cuatro de las siete Uniones de Ejidos que operan en el estado y en el caso de los Fondos de Solidaridad para la Producción, se entrevistó directamente a los presidentes municipales 
de los 16 municipios que cubría el programa en 1992, quienes fungieron como presidentes de los comités de Validación y Seguimiento.

Finalmente, se entrevistaron a los 33 presidentes municipales del estado, siguiendo una guía cuestionario. Estas entrevistas tuvieron la finalidad de captar el papel de la autoridad municipal en el desarrollo del Programa Nacional de Solidaridad, sus actitudes y expectativas, así como los problemas y beneficios que el programa tuvo en ese nivel de gobierno.

\section{Bibliografía}

Anguiano, María Eugenia y Gerardo M. Ordóñez Barba (1994), "Pronasol en Baja California: negociación política y eficacia operativa", El Cotidiano, núm. 62, pp. 69-74.

Bertranou, Julián F. (1993), "Programa Nacional de Solidaridad ¿un nuevo o un viejo modelo de política?", Revista Mexicana de Sociología, núm. 3.

Chávez Galindo, Ana María, David Moctezuma Navarro y Francisco Rodríguez Hernández (1993), "Evaluación del impacto social de Solidaridad en el estado de Morelos", informe final de investigación, México, Centro Regional de Investigaciones Multidisciplinarias (CRIM), unAM/Secretaría de Desarrollo Social (mimeo.).

—- David Moctezuma Navarro y Francisco Rodríguez Hernández (1994), El combate a la pobreza en Morelos; aciertos y desaciertos de Solidaridad, México, CRIM, UNAM.

__ y Francisco Rodríguez Hernández (1993), "Desarrollo regional en el estado de Morelos: el impacto del Programa Nacional de Solidaridad", trabajo presentado en el Seminario Nacional sobre Alternativas para la Economía Mexicana, México, Instituto de Geografía, unam, 26-27 de octubre.

Dresser, Denise (1992), "Pronasol: los dilemas de la gobernabilidad", El Cotidiano, núm. 49, pp. 49-57.

Peón Escalante, Fernando (1992), "Solidaridad en el marco de la política social", El Cotidiano, núm. 49, pp. 14-19.

Programa Nacional de Solidaridad (1991), Evaluación, México, Secretaría Técnica Adjunta de Investigación y Análisis, Comité Técnico de Evaluación del Programa Nacional de Solidaridad.

- (1992), La solidaridad en el desarrollo nacional, México, Secretaría de Desarrollo Social.

Whitehead, Lawrence (1980), "Por qué México es casi ingobernable", Revista Mexicana de Sociología, vol. 42, núm. 1, pp. 203-234.

Zárate, G., Ricardo, Alejandro García G. y Miguel Orozco O. (1993), "Gasto social en México: ¿sacrificios compensados?”, Economia Informa, núm. 220, pp. 47-52. 